\title{
La Fonología del Foco Contrastivo en la variedad de inglés denominada RP y español de Chile
}

\author{
Mauricio Véliz C.*
}

\section{Resumen}

El presente trabajo procura determinar y comparar los mecanismos entonacionales utilizados para establecer contraste en inglés RP y español de Chile. Para este fin, se han empleado corpora de habla espontánea del español de Chile y de la variedad RP del inglés. Los enunciados contrastivos fueron sometidos a análisis acústico, empleando un software especializado y el modelo de fonología entonacional Métrico Autosegmental. Las conclusiones más sobresalientes son las siguientes: (i) la marcación prosódica de contraste aparece como un rasgo mayormente predominante en inglés RP que en español chileno; (ii) el español presenta dos patrones que ocurren con cierta frecuencia: $\left(\mathrm{H}^{*}+\mathrm{L}\right)$ y $\left(\mathrm{L}+\mathrm{H}+, \mathrm{L}^{*}+\mathrm{H}\right)$; sin embargo, en inglés, el uso de $\left(\mathrm{H}^{*}\right)$ sobrepasa ampliamente en número los otros patrones también detectados y (iii) en inglés el uso de $\left(\mathrm{H}^{*}\right)$ se utiliza en más del $50 \%$ de los casos detectados.

Palabras clave: Foco, foco contrastivo, pico tonal, acento tonal, patrón entonacional.

\begin{abstract}
This paper attempts to determine and contrast the intonational mechanisms used in RP English and Chilean Spanish to mark contrastiveness. To this end, corpora of spontaneous speech have been used. The utterances were acoustically analysed, making use of the Autosegmental-Metrical model of intonational phonology. Some of the conclusions are: (i) Contrastivity comes across as a more predominant feature in the English language than in Spanish; (ii) Spanish presents two patterns which occur more often in cases of contrastivity: $\left(\mathrm{H}^{*}+\mathrm{L}\right)$ and $\left(\mathrm{L}+\mathrm{H}^{*}, \mathrm{~L}^{*}+\mathrm{H}\right)$. In English, however, the use of $\left(\mathrm{H}^{*}\right)$ greatly outnumbers the other patterns observed in the corpus; and the use of $\left(\mathrm{H}^{*}\right)$ occurs in more than $50 \%$ of the utterances analysed.
\end{abstract}

Key words: Focus, contrastive focus, peak accent, pitch accent, intonation pattern.

* Coordinador Académico de pedagogía en Inglés de la Universidad Católica Silva Henríquez mveliz@ucsh.cl 


\section{Revisión de la literatura}

Dentro de las áreas que la fonética comprende, la entonación se presenta como una que encierra un sinnúmero de problemáticas que esperan ser abordadas científicamente. En este sentido, uno de los asuntos no resueltos totalmente es el que se refiere a la universalidad o especificidad de los usos lingüísticos de los patrones entonacionales. A este respecto, por ejemplo, O'Connor y Arnold (1973) arguyen que los patrones entonacionales son significativos, sistemáticos y específicos de las lenguas.

Otra área que ha despertado considerable interés, y que constituye la principal motivación de este estudio, es aquella relacionada con lo que Crystal (2001) define como función informacional, de acuerdo a la cual se establece una correlación entre sílabas prominentes e información nueva; correlación que constituye parte central del denominado Modelo Focal en entonación. De acuerdo a este modelo, la información es tratada como 'nueva' o 'dada'. Es en este sentido que Cruttenden (1997:81) presenta muy asertivamente la correlación antes mencionada, estableciendo lo siguiente:

'in many cases (though not all) the focal part of an intonation group can be said to involve new information'.

Al seguir la escuela británica de entonación, de acuerdo a la cual el habla puede ser dividida para propósitos de análisis prosódico en grupos tonales (=grupos entonacionales, unidades tonales, grupos de sentido, etc.), el 'acento nuclear' es frecuentemente asociado con la marcación de foco, información nueva y/o elementos informativos sobresalientes. Halliday (1970:40), en particular, ofrece una explícita descripción de la función textual del 'acento nuclear' antes mencionado, estableciendo lo siguiente:

'the function of the tonic is to form the focus of the information: to express what the speaker decides to make the main point or burden of the message'

La literatura en torno a los elementos constitutivos del grupo tonal, particularmente del acento nuclear, es abundante en descripciones y definiciones cuyos fundamentos van desde la perspectiva puramente fonética, pasando por otras textuales, funcionales, hasta otras cuyas consideraciones se basan en la posición del acento dentro del grupo tonal. En nuestro caso, como ya hemos observado, aquella que liga elementos textuales-funcionales es la más pertinente al momento de indagar en la fonología del foco contrastivo.

Se puede colegir, a partir de los elementos disgregados en los párrafos anteriores, que el análisis de la fonología del foco contrastivo toca temas relacionados con la noción de foco, estructura de la información, acentuación y desacentuación, entre otros. 


\subsection{Foco}

El término foco se refiere, según Bolinger (1972), a un elemento que se destaca en el discurso o que es puesto en un primer plano (=foregrounded), convirtiéndose de esta manera en el elemento más sobresaliente del enunciado. Ladd (1979) acotó el concepto de foco estableciendo que éste puede ser amplio o estrecho, en donde el primero se da cuando todo el enunciado se encuentra en foco, mientras que el segundo ocurre cuando se trata de sólo una porción del enunciado. Ladd (1980) utiliza un ejemplo tomado de Halliday (1967) que ilustra foco amplio:

\section{(1) John painted the SHED yesterday}

En el ejemplo anterior, el acento nuclear cae en shed, lo que correponde a la versión no marcada (=foco amplio) en respuesta a la pregunta: ¿Qué pasó? En el ejemplo, todo el enunciado está constituido por elementos informativos igualmente nuevos.

Foco estrecho puede corresponder, por otra parte, a un ítem léxico/estructural o a una frase gramaticalmente compleja que se destaca a expensas del resto del enunciado. Con el objeto de ilustrar dicha afirmación, Ladd (1980) proporciona ejemplos tomados de Halliday (1967):

(2) JOHN painted the shed

\section{(3) John painted the shed YESTERDAY}

En el ejemplo (2) John se encuentra en foco, puesto que se realizó, seguramente, en respuesta a preguntas tales como: Who painted the shed yesterday?, es decir, una pregunta centrada en quién ejecutó la acción de pintar. De la misma manera, en el ejemplo (3) sólo yesterday se encuentra en foco -como en respuesta a la pregunta: When did John paint the shed?, en donde sólo el adverbial de 'tiempo-cuándo' se presenta como nuevo al oyente.

Se hace necesario puntualizar que no todo el material que se encuentre en foco estrecho corresponde necesariamente a material contrastivo; foco estrecho podría también corresponder a elementos de aclaración o simples adiciones informativas, las cuales frecuentemente se realizan como más prominentes. En otras palabras, se puede decir que el foco contrastivo es un tipo de foco estrecho.

\subsubsection{Foco contrastivo}

Tanto el foco amplio como el foco contrastivo comparten un rasgo que se traduce en destacar una porción del enunciado. El foco amplio introduce información asumida como ausente en la conciencia del interlocutor (Chafe, 1974). En el mismo sentido, Cruttenden (1997:82) define foco contrastivo como:

'involving comparison within a limited set' 
Cabe destacar, sin embargo, que la definición proporcionada por Cruttenden podría conducir a razonamientos engañosos, puesto que los elementos de un enunciado podrían asumirse como tomados de un grupo limitado de ítemes equivalentes. Taglicht (1982) entrega una definición en la que establece que el foco contrastivo ocurre como un par de opuestos; sin embargo, existe un número significativo de enunciados en foco estrecho en donde los elementos que se contrastan muy difícilmente podrían ser considerados como opuestos. Gussenhoven (1999) prefiere referirse al foco contrastivo como corrective (=correctivo), en donde se opta por la marcación de un constituyente que rechaza el uso de otro alternativo, ya sea enunciado por el mismo hablante o por el oyente. La postura de Gussenhoven se puede ilustrar en el siguiente esquema: No es A, sino B.

\subsection{El modelo de fonología entonacional Métrico Autosegmental}

Gran parte del trabajo desarrollado en la última década ha hecho uso de la teoría Métrico Autosegmental de fonología entonacional. Ésta comenzó en la década de los 80 con Pierrehumbert (1980). Se denomina autosegmental puesto que, como dice Face (2002:6): 'tones are phonological units [...] that are independent of, yet associate with, other phonological units'.

Uno de los beneficios más significativos al hacer uso de este modelo está dado por la capacidad de analizar contornos entonacionales altamente complejos en base a dos tonos fonológicos: (i) un tono alto (H) y (ii) un tono bajo (L). Dicho modelo ha demostrado sus virtudes en el análisis de una amplia gama de tipos de lenguas que van desde el chino mandarín hasta el sueco, pasando naturalmente por el inglés y español.

El sistema de marcación utilizado en este modelo ha sido codificado en un grupo de convenciones llamado: ToBI (abreviatura que en inglés representa Tone and Break Index), en el que se marcan dos tipos de acentos: (i) acentos tonales y (ii) acentos de frontera, en donde se asume que el primero (un acento tonal) es un tono -o una secuencia de tonos-asociados a una sílaba acentuada, mientras que el segundo (acento de frontera) se encuentra asociado fonológicamente a las fronteras de frase. Los acentos tonales se marcan convencionalmente con un asterisco (*), el que representa la ubicación real del mismo en la palabra.

Se puede distinguir 7 tipos de acentos tonales:

(i) $\mathrm{H}^{*}$ : Pico tonal: La sílaba acentuada se encuentra en la mitad o en la parte superior del rango tonal del hablante

(ii) L*: Acento tonal bajo: La sílaba acentuada se encuentra en la parte inferior del rango tonal del hablante

(iii) $\mathrm{L}^{*}+\mathrm{H}$ : El tono bajo en la sílaba acentuada es seguido por un ascenso hacia la mitad o parte superior del rango tonal del hablante 
(iv) $\mathrm{L}+\mathrm{H}^{*}$ : El pico tonal es inmediatamente precedido por un ascenso desde la parte baja del rango tonal del hablante; sin embargo L no es tan bajo como en (iii)

(v) $\mathrm{H}+\mathrm{L}^{*}$ : Un valle en la sílaba acentuada es precedido por una caída proveniente de la parte alta del rango tonal del hablante

(vi) $\mathrm{H}^{*}+\mathrm{L}$ : Una caída de una sílaba acentuada en pico tonal seguida de un valle

(vii) ! $\mathrm{H}^{*}$ : La sílaba acentuada se encuentra en la mitad o parte superior del rango tonal del hablante, pero se encuentra más debajo de un $\mathrm{H}^{*}$ precedente

Así como explica Ortiz-Lira (1999), existen dos tipos de asociados a las fronteras entonacionales: (i) acento de frase, ubicado a la derecha del último acento tonal y marcado (-) después del tono H o L y (ii) tono de frontera; ubicado al final de la frase entonacional y marcado con una $\mathrm{H}$ o L, y seguido del símbolo (\%). De lo anterior se desprende que las posibles combinaciones a nivel de fronteras son las siguientes: (i) L-L\%, (ii) L-H, (iii) H-H\% y (iv) H-L\%

\subsection{La entonación del foco contrastivo en español e inglés}

Gran parte del trabajo relacionado con los mecanismos marcadores de contraste en español se ha centrado en mecanismos morfosintácticos (Bolinger 1954; Contreras 1978). De hecho, Ladd (1996:178) establece muy explícitamente lo siguiente:

'while the Roman languages do not allow direct deaccenting, they all have a number of morphosyntactic strategies for achieving similar effects'.

Algunos de los mecanismos morfosintácticos descritos en la literatura incluyen: (i) uso de pro-formas, (ii) topicalización, (iii) frontalización y (iv) clefting.

No obstante, el presente estudio tiene como por objetivo establecer cómo se utiliza la entonación como mecanismo marcador de contraste -área en la que ya ha habido exploraciones interesantes sobre el español (Face, 2002; de la Mota, 1995; García-Lecumberri, 1995; Ortiz-Lira, 1994; Hualde, 1999; Nibert, 2000).

Dentro de los mecanismos entonacionales descritos en la literatura, el tono se destaca considerablemente por sobre la duración silábica u otros rasgos de prominencia. Chafe (1974) asocia tonos bajos a información dada y tonos altos a la información nueva. Además, afirma que los elementos contrastivos pueden tener un tono más alto que aquellos que expresan información nueva. Face (2002) describe tres mecanismos entonacionales que se utilizan a nivel local para expresar contraste:

(i) Se puede usar picos tonales para expresar contraste

(ii) Se puede utilizar H- después de la palabra en foco

(iii)No se observa marcación entonacional en posición final 


\subsection{Acentuación y desacentuación}

En habla espontánea, los hablantes se enfrentan permanentemente a un proceso de acentuación y desacentuación. Uno de los enfoques que intenta dar cuenta de tal proceso es el ya aludido Modelo Focal, en donde Maidment (1990:26) sugiere que foco puede ser concebido como dispositivo general que direcciona la atención, y que responde a decisiones tomadas por los hablantes al tratar la información como nueva o dada, amplia o estrecha. A pesar de que generalmente se relaciona a la información nueva con la acentuación, mientras que la información dada con la desacentuación existen estudios que señalan que es posible encontrar reacentuación de ítemes repetidos (Crystal,1975; Vanderslice \& Pearson, 1967; Cruttenden, 1993). En lo que respecta al español de Chile, Ortiz-Lira (1994) señala que el español utiliza más mecanismos indicadores de foco que el inglés, particularmente de orden sintáctico. Dentro de los medios descritos por Ortiz-Lira (1994) se encuentra el uso de un acento pre-nuclear sostenido, seguido por un núcleo descendente, por ej.
(4) A:
¿Vinieron todos?
B: $\quad$ Ra'quel , vino

\section{El estudio}

El presente estudio aborda el siguiente problema: ¿cuáles son los patrones entonacionales utilizados en inglés $\mathrm{RP}^{1}$ y español de Chile en la marcación de contraste?

Con el fin de resolver el problema antes mencionado, el investigador recogió corpora del español de Chile e inglés RP; el de español comprende tres horas de material grabado, y contiene entrevistas personales y mediales. Los hablantes que toman parte de este corpus son, principalmente, profesionales de grupos etarios diversos. Se debe señalar, además, que el corpus corresponde a una porción de un corpus mucho más amplio recogido para el proyecto Fondecyt 197/1053. Por otra parte, el corpus del inglés está conformado por una colección de entrevistas de audio bajadas desde el sitio Web de BBC Radio 4. Las entrevistas fueron seleccionadas sobre la base dialectal de los participantes (RP o variedad cercana) y la calidad de sonido. Al igual que en el corpus del español, los participantes corresponden a profesionales de grupos etarios variados.

El procedimiento consistió el leer las transcripciones del material grabado, del cual se extrajeron aquellos casos que presentaban contraste léxico o gramatical. Posteriormente, dichos casos fueron analizados fonética y fonológicamente. Los enunciados (22 en total; 11 en cada idioma) fueron sometidos

1 RP es una sigla empleada en inglés (Received Pronunciation) para referirse a una variedad de inglés tradicionalmente asociada a las clases altas del Reino Unido y hablado por una minoría que no supera el 3\%; no obstante ello, es un acento ampliamente enseñado fuera de los confines del Reino Unido. 
a análisis acústico haciendo uso del programa PRAAT 4.1, diseñado por Paul Boersma y David Weenink de The Institute of Phonetics Sciences of the University de Amsterdam. Dicho programa proporciona trazos que muestran las Frecuencias Fundamentales, es decir, la frecuencia más baja en la que un sonido vibra.

\subsection{Objetivos}

Los objetivos de este estudio son los siguientes:

(i) Determinar y contrastar los mecanismos entonacionales utilizados en inglés RP y español de Chile

(ii) Proporcionar antecedentes sobre la universalidad (o especificidad) de los diversos patrones entonacionales utilizados en la marcación de contraste

(iii)Describir e ilustrar las manifestaciones prosódicas del foco contrastivo

\subsection{Hipótesis de trabajo}

Se utilizan picos altos acentuales $\left(\mathrm{H}^{*}\right)$-con acentos tonales precedentes más bajos, si corresponde- para expresar contraste en más de 50\% de los enunciados analizados, tanto en inglés RP como en español chileno.

\section{Análisis}

Siguiendo la teoría de Lambrecht (1994:43) con respecto al tratamiento de la información, se debe señalar que ésta no debería ser asociada automáticamente con largos segmentos de habla; un solo ítem 'influences the hearer's mental representation of the world'. Esto, puesto que en la presente investigación, se encontró que tres morfemas atrajeron acento contrastivo nuclear: dos prefijos que denotan relaciones oposicionales (valid vs. INvalid y advantage vs. DISadvantage), además del sufijo utilizado para propósitos de comparación (safe vs safER). Como se puede observar, sólo en inglés se encontraron ejemplos en donde un morfema cumple la función contrastiva léxica y fonética, o que probablemente se debe a una eventual mayor reacentuación en español que en inglés- tal como lo señala García-Lecumberri (1995).

Por otro lado, los contrastes encontrados en los corpora corresponden a casos en donde el contraste propiamente tal se da en torno a tiempos gramaticales de un mismo verbo, formas verbales, objetos, preposiciones, pronombres, morfemas, frases preposicionales, etc.

Las unidades contrastivas han sido etiquetadas Al, cuando éstas son introducidas por primera vez, y A2 con se produce el contraste propiamente tal. Las unidades etiquetadas B corresponden a material post-contrastivo, el que normalmente contiene material léxico repetido. Los enunciados han sido divididos en dos partes; sin embargo, se presenta la versión ortográfica completa de ellos en la parte superior de los trazos. 
Del análisis del corpus se puede señalar que de los 11 enunciados que presentaban contraste léxico en español, dos de ellos no evidenciaron un contraste fonético en las unidades contrastivas. Se evidencia, por lo tanto, en estos casos el fenómeno de la reacentuación. Sin embargo, estos casos podrían ser igualmente interpretados -así como lo señala Ortiz-Lira (1994)- como un mecanismo propio del español para expresar contraste por ej.

(5) Porque ella no era actriz y ahí yo la hice actriz

$$
\text { A1 A2 }
$$

...Y ahí yo la hice actriz.

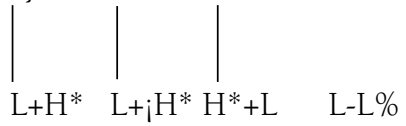

El corpus del inglés, por su parte, presenta un mayor grado de desacentuación de elementos B que el corpus del español. Dichos elementos corresponden a material dado en posiciones post-contrastivas, por ej.

(6) And simply it wasn't true when quite clearly it was true.
Al B1
A2 B2
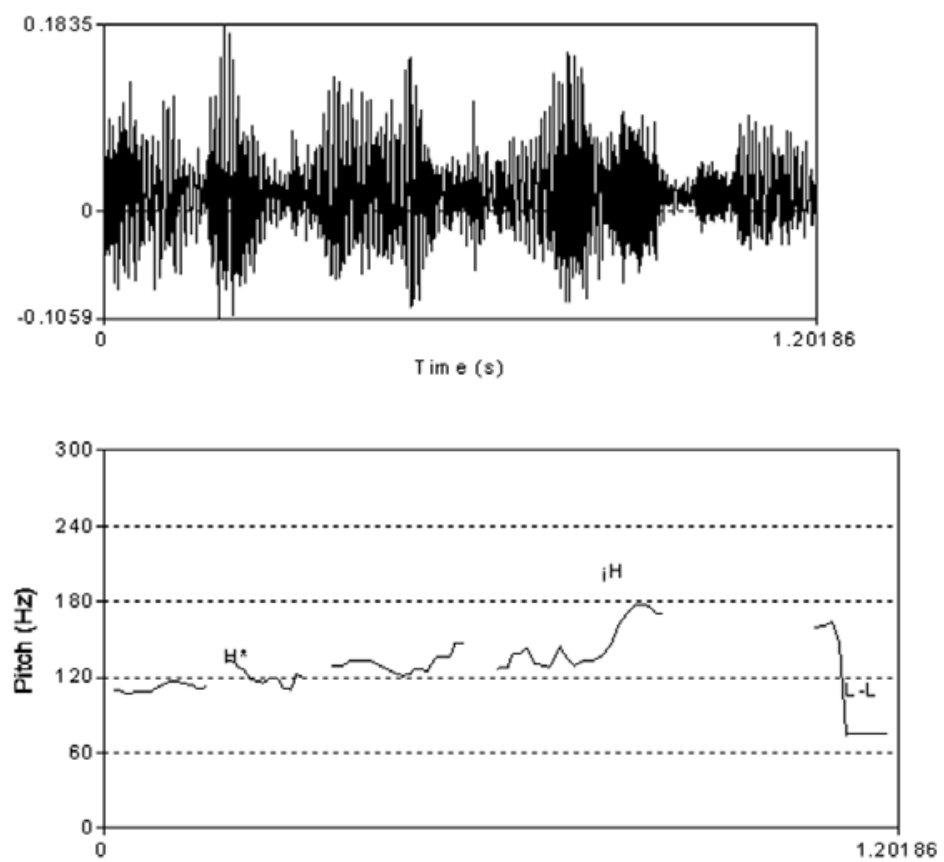
When quite clearly it was true

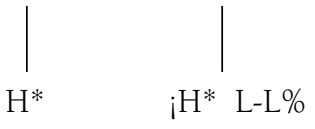

(7) No podía seguir estudiando, o no podía empezar a estudiar.

$\begin{array}{llll}\text { A1 } & \text { B1 } & \text { A2 } & \text { B2 }\end{array}$
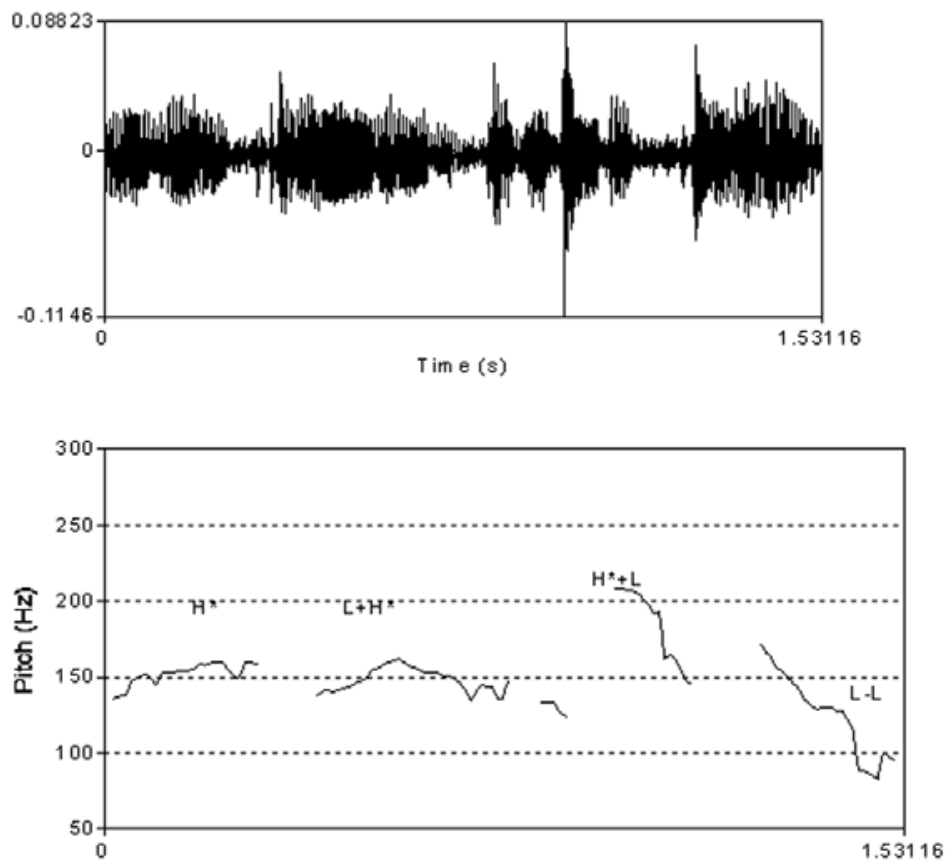

... o no podía empezar a estudiar

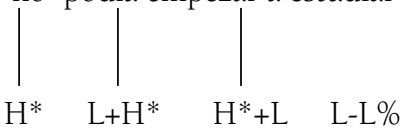

\subsection{Realizaciones de contraste en español}

El corpus del español demuestra que existen dos patrones que tienden a ser favorecidos por los hablantes: (i) el patrón $\left(\mathrm{H}^{*}+\mathrm{L}\right)$ (ver 7), con tres ejemplos y (ii) $\left(\mathrm{L}+\mathrm{H}^{*}\right)$, con otros tres ejemplos. En ambos casos, los acentos nucleares contrastivos son precedidos por $\left(\mathrm{H}^{*}\right)$ o $\left(\left(\mathrm{L}+\mathrm{H}^{*}\right)\right.$. Los patrones de realizaciones menos frecuentes corresponden a $\left(\mathrm{L}^{*}\right)$ y $\left(\mathrm{H}^{*}\right)$, con sólo dos ejemplos el primero y uno el segundo. 
El siguiente gráfico ilustra los diversos patrones encontrados en el corpus de español.

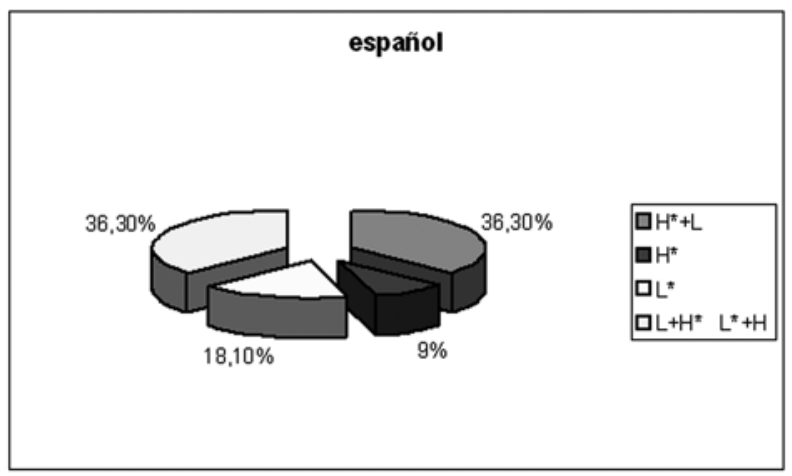

\subsection{Realizaciones de contraste en inglés}

El análisis del corpus del inglés reveló que en más de la mitad de los enunciados se utiliza $\left(\mathrm{H}^{*}\right)$ para expresar contraste. Todos los ejemplos-salvo 1- presentan un acento tonal precedente, el que en varios casos fue bastante bajo, lo que permitió visualizar un escalamiento (upstep) (ver 6). El otro patrón, presente en sólo dos enunciados, corresponde al uso de $\left(\mathrm{L}+\mathrm{H}^{*}\right)$.

El siguiente gráfico muestra los patrones entonacionales encontrados en el corpus del inglés.

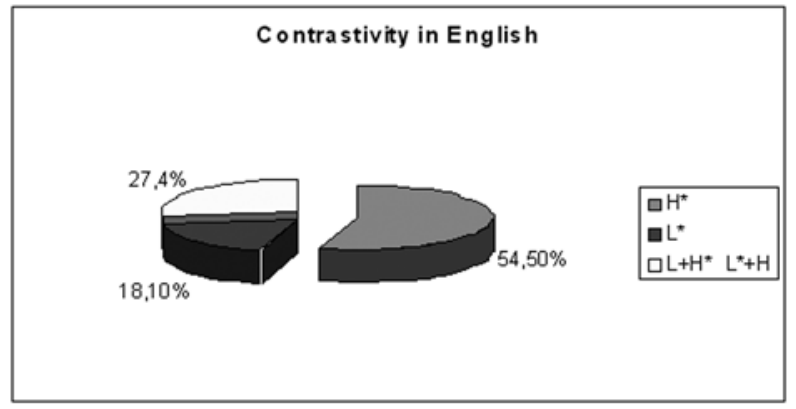

\section{Conclusiones}

Coincidentemente resultó que después de la búsqueda de casos que presentaran contraste léxico-gramatical y subsecuente análisis fonético y fonológico, se obtuvo el mismo número de enunciados que eventualmente conformaron los corpora. Dicha coincidencia permitió, dentro de las limitaciones que implican corpora considerablemente enjutos, establecer conclusiones preliminares simétricas. Éstas se presentan a continuación: 
i) Los corpora de ambas lenguas revelaron que las unidades contrastivas (A2 en este trabajo) tienden a ocurrir en posición media o final, con sólo un ejemplo en cada corpus en posición inicial. Además, se observó que los patrones entonacionales empleados para expresar contraste son bastante variados, lo que nos hace concluir que no existe una correlación directa entre un patrón entonacional determinado y la posición del elemento contrastivo.

ii) La marcación prosódica de contraste aparece como un rasgo mayormente predominante en inglés RP que en español chileno, puesto que en el primero los hablantes optan por marcar contraste en cualquier elemento que denote contraste, a saber prefijos, sufijos, morfemas usados para expresar contraste, etc. En consecuencia, la desacentuación de material dado en inglés ocurre en todos los casos que presentan elementos B2, mientras que en español existen dos ejemplos que presentan reacentuación de información dada.

iii) El español presenta dos patrones que ocurren con cierta frecuencia: $\left(\mathrm{H}^{*}+\mathrm{L}\right)$ y $\left(\mathrm{L}+\mathrm{H}+, \mathrm{L}^{*}+\mathrm{H}\right)$. Sin embargo, en inglés, el uso de $\left(\mathrm{H}^{*}\right)$ sobrepasa ampliamente en número los otros patrones también detectados. Un patrón coincidente encontrado en ambos corpora corresponde a la combinación de un ascenso y descenso.

iv) Se observó, además, que el escalamiento (traducción directa del término upstep del inglés) es un rasgo que se presenta con más frecuencia en inglés. En español, sin embargo, no parece existir un patrón claramente definido con respecto al material pre-contrastivo.

v) $\left(\mathrm{L}^{*}\right)$ es empleado de manera igualmente ocasional en ambas lenguas.

vi) A pesar de algunas similitudes encontradas en los corpora de las dos lenguas aquí analizadas, se advierte aún la necesidad de corroborar las tendencias presentadas en este trabajo, haciendo uso de corpora de mayor volumen.

vii) Finalmente, la hipótesis se muestra parcialmente validada, puesto que en inglés el uso de $\left(\mathrm{H}^{*}\right)$ se utiliza en $54 \%$ de los casos.

\section{Bibliografía}

Bolinger, D. L. (1954). 'English prosodic stress and Spanish sentence accent'. Hispania, 37, 152-6.

Bolinger, D. L. (1972). 'Accent is predictable (if you're a mind reader)'. Language, 48, 633-44.

Bolinger, D. L. (1986). Intonation and its parts: Melody in spoken English. Stanford, Calif.: Stanford University Press.

Chafe, W. (1974). Meaning and the structure of language. Chicago: University of Chicago Press.

Contreras, H. (1978). El orden de palabras en español. Madrid: Cátedra. 
Cruttenden, A.(1993). 'The De-accenting and Re-accenting of repeated lexical items'. In House, D. \& Touati, P. (eds.), Proceedings of an ESCA workshop on prosody. Working Papers, 41, 16-19.

Cruttenden, A. (1997). Intonation. Cambridge: Cambridge University Press.

Crystal, D. (1975). The English tone of voice: Essays in intonation, prosody and paralanguage. London: E. Arnold.

Crystal, D. (2001). The Cambridge encyclopedia of the English language. Cambridge: Cambridge University Press.

de la Mota, C. (1995). La representación gramatical de la información nueva en el discurso. Unpublished doctoral dissertation, Universitat Autonoma de Barcelona.

Elsner, A. (1994). Distinction between 'normal' and 'contrastivelemphatic' focus. Bonn: University of Bonn.

Face, T. (2002). Intonational marking of contrastive focus in Madrid Spanish. Munich: Lincom Europa.

García-Lecumberri, M. (1995).

Intonational signalling of information structure in English and Spanish: A comparative study. Unpublished doctoral dissertation, University of London.

Garrido, J. (1996). Modelling Spanish intonation for text-to-speech applications. Unpublished doctoral dissertation, Universitat Autónoma de Barcelona.

Gussenhoven, C. (1999).

Types of focus in English. The Netherlands: University of Nijmegen. Retrieved October 4, 2004 from World Wide Web: http://www.let.kun.nl/ C.Gussenhoven/Matt.pdf

Halliday, M. A. K. (1967).

Intonation and grammar in British English. The Hague: Mouton.

Halliday, M. A. K. (1970).

A course in spoken English: intonation. London: Oxford University Press.

Hualde, J. (1999). "Basic intonational contours in Spanish". Paper presented at the first Sp-ToBI workshop, Columbus, Ohio, 1-3 October.

Ladd, D.R. (1979). 'Light and Shadow: A study of the syntax and semantics of sentence in English', in Waugh, L.R. \& Van Coetsen, F. (eds.), Contributions to grammatical studies semantics and syntax. Leiden: E. J. Brill.

Ladd, D. R. (1980). The structure of intonational meaning. Bloomington: Indiana University Press.

Ladd, D. R. (1990). $\quad$ Review article: 'Intonation vs. grammar'. Review of D. L. Bolinger (1989). Intonation and its uses, Language, 66, 806-16. 
Ladd, D. R. (1996). Intonational phonology. Cambridge: Cambridge University Press.

Lambrecht, K.(1995). Information structure and sentence form. Cambridge: Cambridge University Press.

Maidment, J. (1990). 'Focus and tone in English intonation'. In S. Ramsaran (ed.), Studies in the pronunciation of English. London: Routledge.

Navarro, T. (1918). Manual de pronunciación española. Madrid: Centro de Estudios Históricos.

Navarro, T. (1944). Manual de entonación española. New York: Hispanic Institute in the United States.

Nibert, H. (2000). Phonetic and phonological evidence for intermediate phrasing in Spanish intonation. Doctoral dissertation, University of Illinois at Urbana-Champaign.

O'Connor, J. D. and Arnold, G. F. (1973).

Intonation of colloquial English. London: Longman.

Ortiz-Lira, H. \& Finch, D. (1982).

A course in English phonetics for Spanish speakers. London: Heinemann.

Ortiz-Lira, H. (1994). A contrastive analysis of English and Spanish sentence accentuation. Unpublished Ph. D. thesis, University of Manchester.

Ortiz-Lira, H. (1999). 'La aplicación de ToBI a un corpus del español de Chile'. Onomázein, 4, 429-442.

Pierrehumbert, J. (1980).

The phonology and phonetics of English intonation. Doctoral dissertation, MIT.

Prieto, P. (1998). 'The scaling of the L values in Spanish downstepping contours'. Journal of Phonetics, 26, 261-82.

Selkirk, E. (2001). C Contrastive focus vs. Presentational focus: Prosodic evidence from right node raising in English. University of MassachusettsAmherst. Retrieved October 10, 2004 from World Wide Web:http://www.lpl.univ-aix.fr/sp2002/pdf/selkirk.pdf

Sosa, J. (1991). Fonética y fonología de la entonación del español hispanoamericano. Doctoral dissertation, University of Massachussets.

Taglicht, J. (1982). 'Intonation and the assessment of intonation'. Journal of Linguistics, 18, 213-30.

Trager, G. L. \& Smith, H. L. (1951).

An outline of English structure. Washington, D. C.: American Council of Learned Societies.

Vanderslice, R. \& Pearson, L. S. (1967).

'Prosodic features of Hawaiian English'. Quarterly Journal of Speech, 53, 156-66. 